\title{
A Fault Tolerant Algorithms for the Minimization of Blocking Probability in Optical Burst Switching Network
}

\author{
Paramjeet Kaur \\ M.Tech (CSE), Indo Global \\ College of Engineering, Abhipur, \\ Mohali- Punjab, INDIA
}

\author{
Hardeep Singh \\ A.P (ECE), Indo Global College of \\ Engineering, Abhipur, \\ Mohali-Punjab, INDIA
}

\author{
Satinder Pal Ahuja \\ A.P (CSE), Indo Global College of \\ Engineering, Abhipur, \\ Mohali-Punjab, INDIA
}

\begin{abstract}
One of the major concerns in the field of computer Network is how to Transfer large amount of data and Transfer that data without any congestion or faults. Optical Burst Switching networks are used today for the huge transfer of data. So, Fault Tolerance is an important Issue in the Optical burst switching network. Fault tolerant refers to the ability of the network to transfer the information at the same amount as if any type of fault occurs in the network. In any network there are number of path to reach from source to the destination so, first the calculation of shortest path must be required. Blocking probability is the parameter which is used for the calculation of the possibility of the particular path to be blocked. In the traffic engineering Engset formula is used for the calculation of the blocking probability in the network there are the finite population sources. This paper includes the designing of new algorithm for fault tolerant and comparison of Algorithm with already existed algorithms. This algorithm is based on the shortest path calculation, checking of the fault on the each path, Calculation of blocking on paths which are fault free then selects the path with the minimum blocking probability.
\end{abstract}

\section{General Terms}

Optical Networks, Congestion, Traffic, Routing, Shortest Path

\section{Keywords}

Optical burst switching network, Shortest path,Blocking probablity,Congestion, Fault tolerance,Engset formula .

\section{INTRODUCTION}

The demands of bandwidth for telecommunication networks are rapidly increasing worldwide due to the increase in number of users in communication networks. Also the new services with higher bandwidth requirements are developing which leads to the data traffic. Therefore, many questions and problems regarding to above are arising like, design \& planning of such networks, performance $\&$ cost evaluation, development of operation \& management concepts etc [1].There are many technologies which are helpful for solving these problems. Optical burst switching (OBS) is the important technology. This technology combines the advantages of both optical circuit switching (OCS) and optical packet switching. OBS is a telecommunication technique that allows dynamic sub-wavelength switching of data.

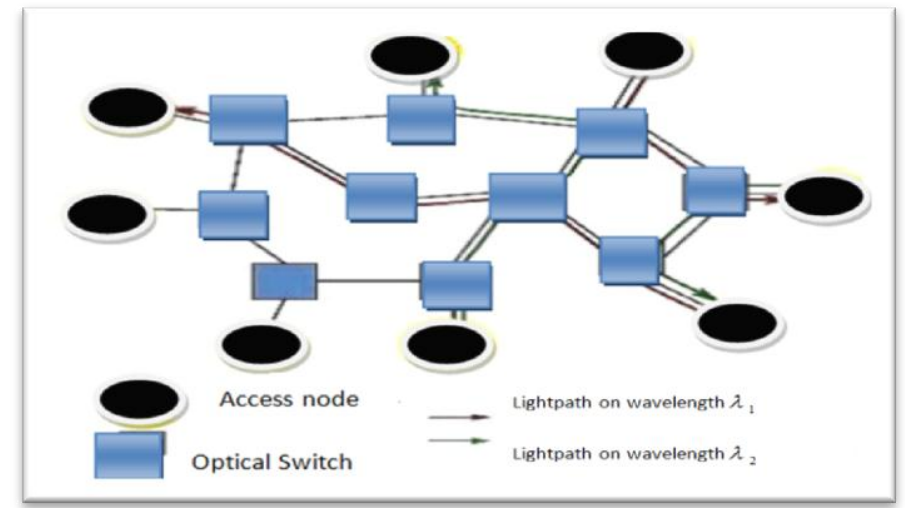

Fig 1:A Wavelength-routed optical WDM network with Lightpath Connections

It is similar to optical packet switching, but basically differs in that the control signals are sent separately and in advance of the data. By doing so, these control signals can be processed electronically and allow the timely setup of an optical light path to switch the soon-to-arrive data, thereby eliminating the need for large optical buffer. The purpose of OBS is to dynamically provision sub-wavelength granularity by optimally combining electronics and optics.

\subsection{Congestion in OBS network}

In data networking and queuing theory, network congestion occurs when a link or node is carrying so much data that its quality of service deteriorates. Typical effects include queuing delay, packet loss or the blocking of new connections. A consequence of these latter two is that incremental increases in offered load lead either only to small increase in network throughput, or to an actual reduction in network throughput.

\subsection{Fault tolerant in OBS Network}

Since each lightpath can carry a huge amount of traffic, failures in such networks may seriously damage end-user applications. According to the scale of their effect, failures in all-optical WDM networks can be classified into two categories [4]. One category is a wavelength-level failure which impacts the quality of transmission of each individual lightpath. The other category is a fiber-level failure which affects all the lightpath on an individual fiber. Fault tolerance schemes can be broadly classified into: 
- Path Protection:-The fault tolerance schemes can be classified into path protection and path restoration. In path protection, backup resources are reserved during connection setup and primary and backup paths are computed before a failure occurs.

-Restoration:- In path restoration, the source and destination nodes of each connection traversing the failed link participate in distributed dynamically discover an end to- end backup route.

\section{TRADITIONAL ALGORITHMS FAULT TOLERANT IN OPTICAL BURST SWITCHING NETWORK}

Various algorithms, for fault tolerant are given below.

- Random Packet Assembly Admission Control (RPAAC) algorithm is a traffic engineering mechanism which monitors the network congestion and proactively drops incoming packets at ingress nodes before they may actually become harmful to the network.

- Wavelength routed optical network This algorithm provides the low call blocking probability and also employ very short computation time. The blocking performance of DRWA algorithm is measured in terms of the mean call blocking probability.

- Novel fault-tolerant node architecture using a resilient buffer. In their model buffer is attached for each outgoing link [17].

\section{- Fault Tolerant Least Congestion algorithm (FTLCA):}

The FTLCA algorithm is basically on congestion on paths between the s-d pairs. The blocking probability mostly occurs due unbalancing of congestion on paths between s-d paths. First algorithm selects the s-d pair, and then calculates the number of available paths between the selected s-d pair. After the calculation of number of available paths, checking of congestion on each path will be done. Then algorithm sorts the values of congestion in increasing order. Normally the path with minimum congestion will offer least blocking probability. On this criterion algorithm selects the first path in order of congestion. After the selection of path, the checking of path for fault that leads to blocking probability. If fault exits then select the second path in order of congestion, otherwise call will be established on selected path.

- Fault Tolerant Optimized Blocking Algorithm (FTOBA):

Similar to FTLCA, the FTOBA is also congestion based but in this algorithm blocking probability on each will be calculated. First algorithm selects the s-d pair, and then calculates the number of available paths between the selected s-d pair. After the calculation of number of available paths, checking of congestion on each path will be done. After calculating the blocking probability for each path, arrange the paths in increasing order of blocking probability. The first path will be selected in order of blocking probabilities. Then algorithm checks the call which is blocked or not, because least blocking, does not mean that there is no fault when we chose the path with least blocking probability at the time of call establishment the call may be blocked due to any fault. If call is blocked then select the next path in the order of blocking probability. If call not blocked then, call is established. And then compares the performance of these algorithms on the basis of blocking probability. These algorithms are based upon the congestion on path in OBS network and based on the simulation results, that the reliable and fault tolerant routing algorithms reduces the blocking probability [2].

\section{PROBLEM FORMULATION}

Optical Burst Switching (OBS) is a technique to support bursty traffic over wavelength-Division- Multiplexed (WDM) networks .WDM offers the capability to handle the increasing demand of network traffic. In Optical Burst Switched networks, each Light path carry huge amount of traffic, path failures may damage the user application. Hence fault-tolerance becomes an important issue on these networks. Many problems exist with the already proposed algorithm in the literature. To overcome these problems this thesis work designing a new algorithm for fault tolerant. It has been observed that the value of blocking is increased with the increased in congestion. The blocking probability is same for the different number of wavelength.

\section{OBJECTIVE FOR STUDY}

- To analyze different algorithm proposed in literature for fault tolerance in OBS networks

- To Design fault tolerance algorithm for optical Brust switching networks for minimization of Blocking Probability.

- To compare the proposed algorithm with the prior algorithm proposed in literature.

\section{PROPOSED FAULT TOLERANT ALGORITHMS}

In this paper a new algorithm is proposed that is fault tolerant algorithm for optimization of blocking probability in OBS network. The objective of this algorithm is to minimize the blocking probability. This analytical model is designed under the following assumptions:

- Optical network is set of node interconnected by sing le-fiber link.

- $\quad$ Each fiber link is bi-directional and each link has $\lambda$ wavelength channel.

- Each Station has array of transmitters and receivers, where $\lambda$ is the wavelength carried by each fiber.

- Number of available wavelength $=1$.

- No, queuing of connection request. If connection is blocked next alternative path is selected.

- Link loads are independent.

- Here non-adaptive alternative path allocation is assumed in this thesis work.

To calculate the blocking probability we will use the Engset formula as in equation (3.1). The Engset formula has been used extensively in the traffic engineering of optical communication. Erlang is defined as dimensionless unit of traffic intensity. It is dependent on observation time. The maximum facility can be in use is $100 \%$ of the time. If the observation time is 10 minutes, and facility is in use for the full time, then that is 1 Erlang.

Steps for the implementation of the algorithm are given below:

Step1:-Select a source and destination in an Optical Burst network through which we want to transfer the data. 
Step2: Define all the routes between the source and destination.

Step3: Apply the Dijkstra algorithm for finding the all possible paths from the source to the destination by calculating the candidate distance from each node that is given by

Candidate distance $=$ distance to the solved node + length of $\operatorname{arc}$

\section{Candidate distance \\ $=$ distance to the solved node + length of arc}

Step4: Arrange all the path or routes from the source to destination in increasing order.

Step5: Select the shortest path.

Step6: Check for the fault on the selected path if fault exist

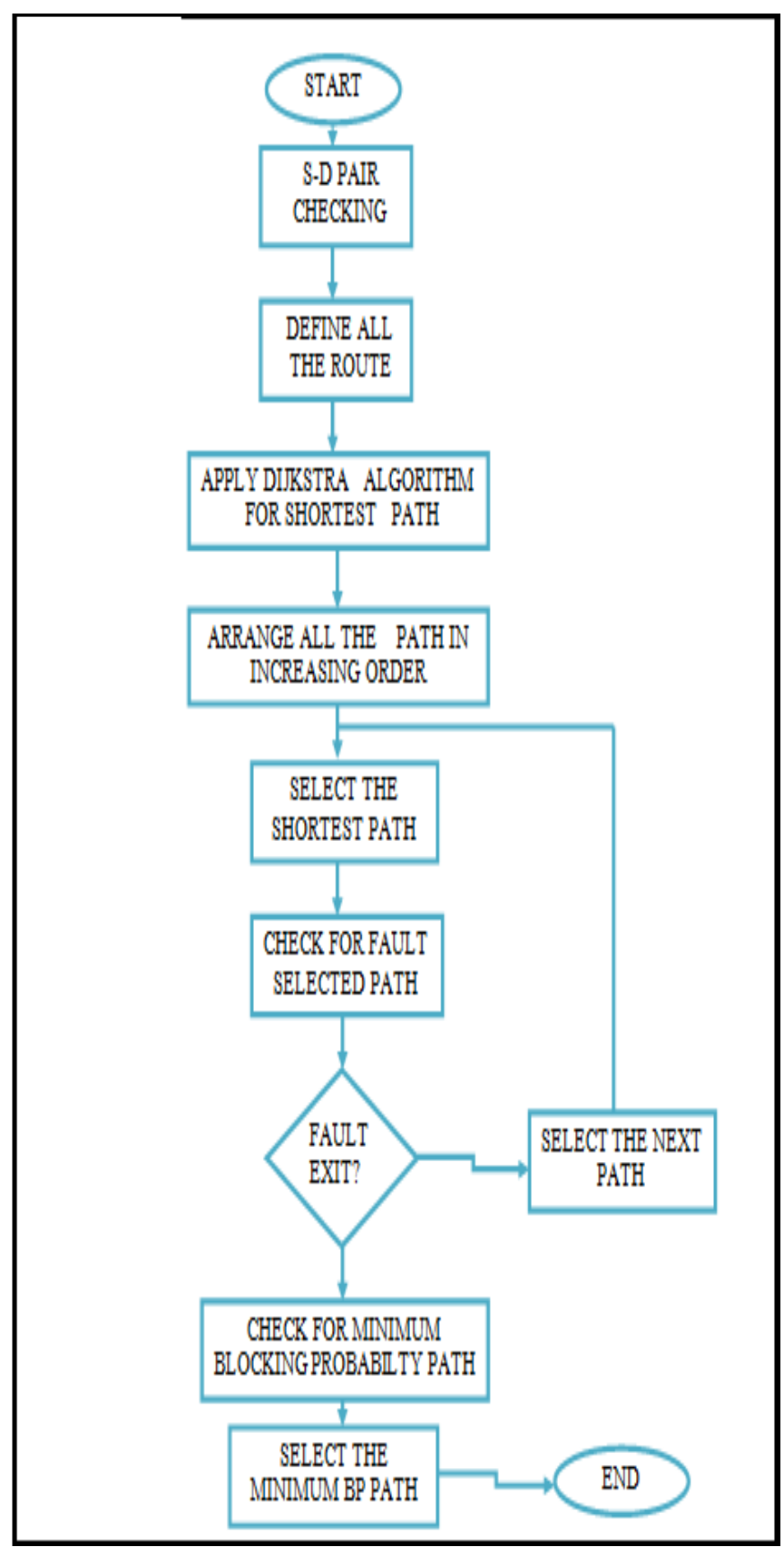

Fig2:flowchart for fault tolerant algorithm on this path select the next path. Calculate the BP on each path using

the Engset formula.

$$
P_{b}(N, A, S)=\frac{A^{N}\left(\begin{array}{c}
S \\
N
\end{array}\right)}{\sum_{i=0}^{N} A^{i}\left(\begin{array}{c}
S \\
i
\end{array}\right)}
$$

Where

$$
\begin{gathered}
A=\text { offered traffic intensity in erlangs, from all } \\
N=\text { number of circuits in group } \\
S=\text { number of sources of traffic } \\
\mathrm{P}(\mathrm{B})=\text { probability of blocking or congestion }
\end{gathered}
$$

Step 6: Check the blocking probability on each path on which there is no fault then arranges the BP of every path in increasing order and select the minimum blocking probability path.

Then flow chart shown in Figure 2 illustrates the mechanism of FAULT TOLARANT ALGORITM FOR MINIMIZATION OF BLOCKING PROBABILTY IN OPTICAL BURST SWITCHING NETWORK.

\section{RESULTS AND DISCUSSION}

The simulation is carried out on simulation software MATLAB 7.5 of Math work. In this section we will compute the results for the different values of number of servers, number of users and traffic intensity. We have fixed the value of number of servers to 30 , number of users to 20 and varied the traffic intensity. We have calculated the results as shown in figures given below.

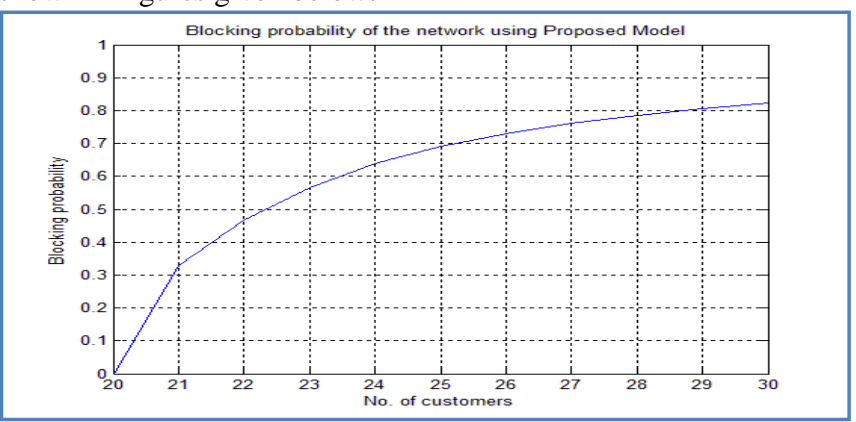

Fig 3: Graph showing the Blocking probability vs. number of customers for $\rho=10$

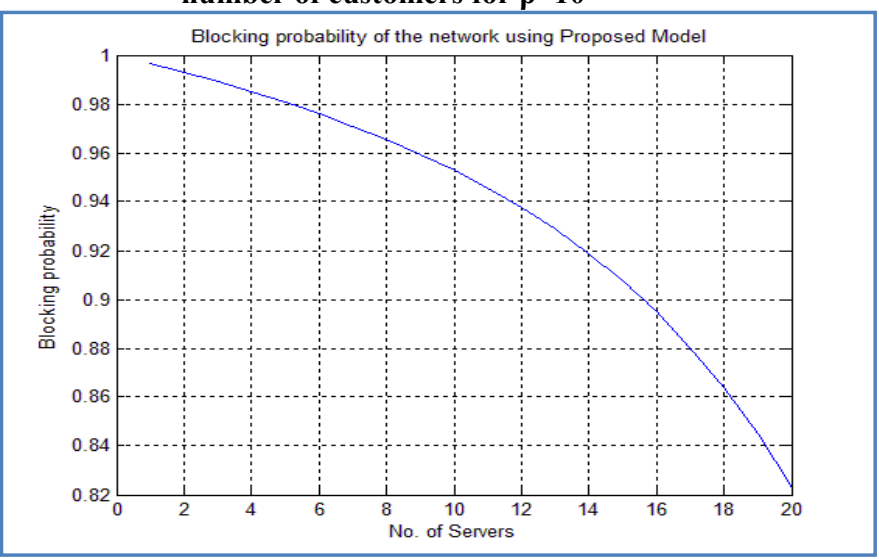

Fig 4: Graph showing the Blocking probability vs. number of servers for $\rho=10$ 


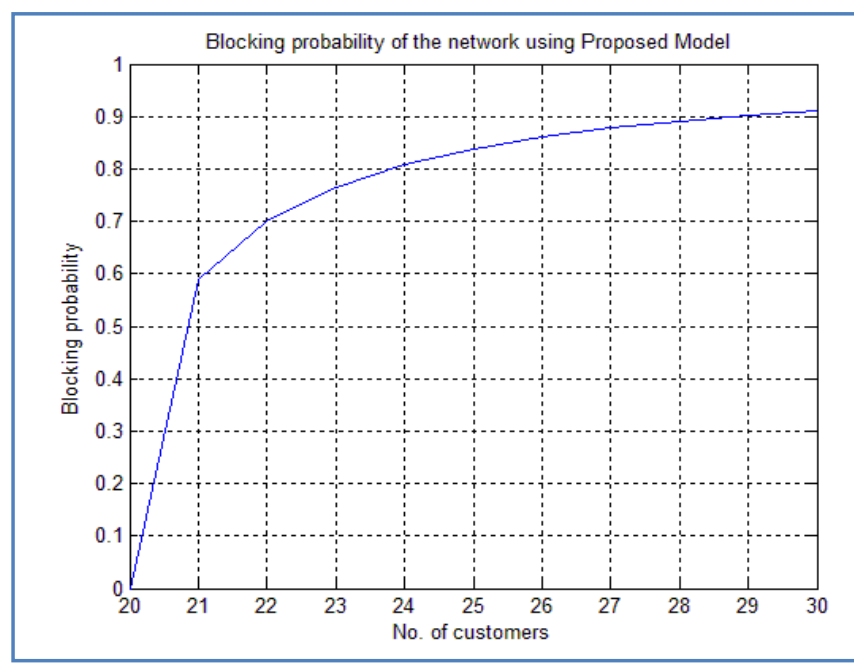

Fig 5: Graph showing the Blocking probability vs. number of customers for $\boldsymbol{\rho}=\mathbf{2 0}$

\subsection{Comparative Implementation}

When we compared these results with the results given in by (Hardeep Singh, Dr.Jai Prakash et al. 2011) we found in figure 8 that the value of blocking probability of the FTOBA algorithm proposed by (Hardeep Singh, Dr.Jai Prakash 2011 et al.) is nearly 0.98 when the maximum value of congestion is fixed to 30 with one wavelength whereas the results shown in figure 7 show that the blocking probability of the system is nearly 0.94 with one wavelength in the proposed model.

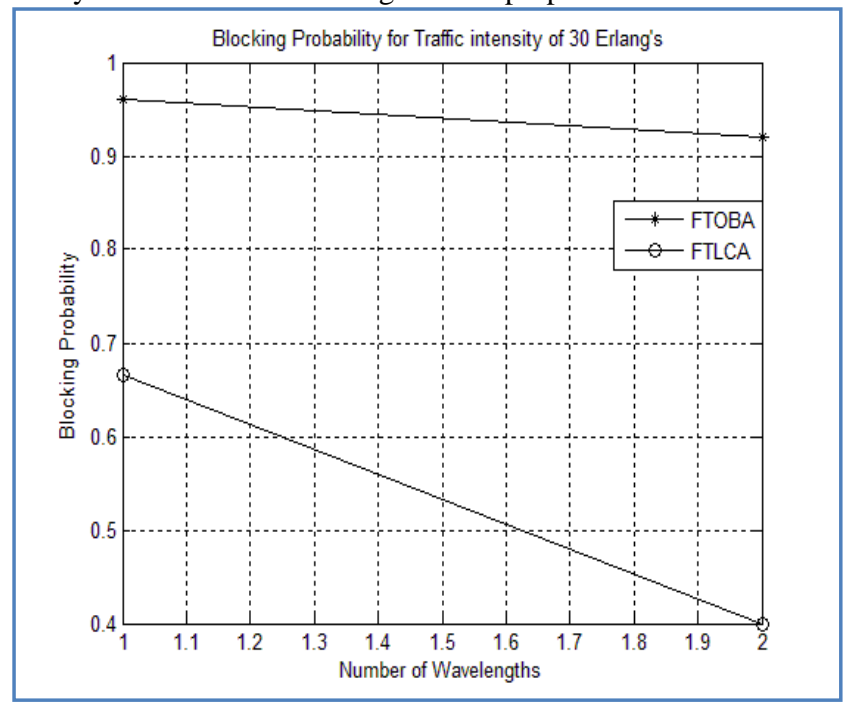

Fig 6: Comparison Graph

Thus we can conclude that the results prove that our proposed model gives Better result than the algorithm proposed by (Hardeep Singh, Dr.Jai Prakash 2011 et al. 2011).

By using the other parameters blocking probability is also decreased

- $\quad \mathrm{BP}=0$ when number of server $=$ number of user

- $\quad$ BP is reduced by the 0.03 in the proposed model

- Blocking probability w.r.t number of customer $=8.1$
- Blocking probability decreased with the increase in number of servers.

\section{CONCLUSIONS}

To objectively assess the degree of blocking probability in Optical Burst Switching network, we applied algorithm for the calculation of routes, randomly detect the fault and after that calculate the blocking probability on each path. The experimental results have shown that our comparison is made on the basis of different parameters like Number of server, number of user, Traffic intensity and the blocking probability. So proposed Algorithm as compare to algorithm that is fault tolerant optimized blocking algorithm proposed by (Hardeep Singh, Dr.Jai Prakash 2011 et al. 2011) shows good results. It reduces the blocking probability in the Optical burst switching network. Experimental evaluation of the performance of Algorithm showed its ability to Reduce the blocking probability when the number of server are more and number of user are also limited according to the number of server. As it is clear from graph results that the if there is a some limitation for number of user and the to some extent to the traffic intensity and it has increased the quality of service of a optical burst switching network. But the blocking probability is not removed totally. In this work we have made comparison between two Algorithms to reduce the blocking probability by using the different number of parameters used in the Optical burst switching network. The Proposed algorithm performs better in comparison to fault tolerant optimized blocking algorithm proposed by (Hardeep Singh, Dr.Jai Prakash 2011 et al. 2011.

\section{FUTURE SCOPE}

In future we can extend our research to work on the different parameters like by enhancing the number of wavelength in the network. As this work can be extended for the infinite number of users. We can further apply new formulas for the minimization of the blocking probability on the different parameters. The proposed algorithm can be implemented on the network simulators.

\section{REFERENCES}

[1] Hardeep Singh, Dr.Jai Prakash, Dinesh Arora, Dr.Amit Wason (Sept. 2011) "Contention Mitigation in Optical Burst Switching using First-Fit, Random and Reserved Channel Scheduling” IJECT Vol. 2, Issue 3.

[2] Hardeep Singh, Dr.Jai Prakash, Dinesh Arora \& Dr.Amit Wason (2011) "Fault Tolerant Congestion Based Algorithms in OBS Network”, (IJE), Volume (5), Issue (5).

[3] Hardeep Singh, Dr. Jai Prakash, Dinesh Arora, Dr.Amit Wason(Nov.2011) "Analysis of Blocking Probability in OBS Network", International journal of sciencetific and engineering research11,volume2,issue 11 .

[4] MD. Shaminreza (2010) "Evaluation of brust rate of an optical burst switching (OBS) network with wavelength conversion capability".

[5] G.Ramesh, S.SundaraVadivelu (2009) "A Reliable and Fault-Tolerant Routing for Optical WDM Networks", (IJCSIS), Vol. 6, No. 2.

[6] D.Callegati, F.Matera, T.Franzl and C. Munoz (2009) "Report on Y2 activities and new integration optical network in Europe", FP7-ICT-216863/DEISUNIBO/ R/PU/D11.2, 2009. 
[7] X.Masip Bruin, M.German, A.Castro, E.Marin-Tordera, R.Serral-Gracia and E. gramping (2009) "The Minimum coincidence routing approach in wavelength-routed optical WDM networks", 978-1-4244-4550-9/09, IEEE 2009.

[8] X.Masip-Bruin, M.German, A.Castro, E.Marin-Tordera, R.Serral-Gracia and E.grampin (2009) "The Minimum coincidence routing approach in wavelength-routed optical WDM networks", 978-1-4244-4550-9/09, IEEE 2009.

[9] J.Zhang, L.Song and B.Mukherjee (2007) "A FaultTolerant OBS Node Architecture with Resilient Buffers", Optical Communication (ECOC) 33rd European Conference and Exhibition, 978-3- 80073042-1, Sep.2007.

[10] L. Kungmeng, D.Habibi, Q.V.Phung and H.N.Nguyen, "Dynamic Wavelength Routing in all-Optical Mesh Network", Asia-Pacific Conference on Communication, Perth, Western Australia,177-182.0- 7803-9132-2/05, IEEE 2005

[11] Dr. Farid Farahmand and Dr. Qiong (Jo) Zhang (2004) "Circuit Switching" Central Connecticut State University and Arizona State University at West Campus Optical Burst Switching Networks, IEEE Communications Society.

[12] Kaheel, A. (2004) “Analytical evaluation of blocking probability in optical burst switching networks"
Communications, 2004 IEEE International Conference, vol.3.

[13] D.Bisbal, I.D.Miguel, F.Gonzalez, J.Blas, J.C.aguado and M. Lopez (2004) "Dynamic Routing and wavelength Assignment in Optical Networks by Means of Genetic Algorithms", Photonic network Communication, Vol.7, No.1, 43-58, 2004.

[14] Zvi Rosberg, Hai Le Vu, Moshe Zukerman and Jolyon White(2003)"Blocking Probabilities of Optical Burst Switching Networks Based on Reduced Load Fixed Point Approximations", IEEE .

[15] Zvi Rosberg, Hai Le Vu, Moshe Zukerman (Sept. 2003) "Performance Analyses of Optical Burst-Switching Networks", IEEE journal on selected areas in communications, vol. 21 , no. 7 .

[16] Yang Chen, Hongyi Wu, Dahai Xu and Chunming Qiao (2003) "Performance Analysis of Optical Burst Switched Node with Deflection Routing" IEEE International Conference on, Volume: 2.

[17] Z.Rosberg, H.L.Vu, M.Zukerman and J.White (2003) "Blocking Probabilities of optical burst switching networks based on reduced load fixed point approximations", 0-7803-7753-2/03, IEEE-2003. 